\title{
Towards Worldview Education beyond Language-Culture Incommensurability
}

International Journal of Science and Mathematics Education

Published online: 29 April 2006; Online Date May 012006

International Journal of Science and Mathematics Education (2007)Vol.5No.1: 29-48

KAWASAKI, Ken

Faculty of Education, Kochi University, 2-5-1, Akebono Cho, Kochi City, 780-8072 Japan

kenknym@aqr.e-catv.ne.jp

\begin{abstract}
This article presents an axiomatic expression of science education: [SCIENCE EDUCATION] is a system of teaching [SCIENCE]. The axiom includes two indefinable terms, [SCIENCE EDUCATION] and [SCIENCE]. In the same way that axiomatics of geometry distinguishes among axioms, postulates and theorems, the axiom presupposes a distinction among the three stages of cognition: axiom, postulate and theorem. This distinction, which is properly called the axiomatics model, will draw science educators' attention to how scientific concepts are distorted, and will develop science education towards worldview education. In the context of science education, worldview education has the potential to enable science educators and pupils to liberate themselves from their language-culture prejudices. On the basis of the axiomatics model, mutual understanding of different language-culture communities will be greatly promoted in the science classroom.
\end{abstract}

Keywords: Language, Worldview, Incommensurability, Axiomatics and Structuralism. 


\section{Introduction}

Recently, I coined the notion of "language mode of science education" for the purpose of examining critically the nature of science education portrayed in non-Standard Average European (SAE ${ }^{1}$ ) language milieus (Kawasaki, 2002). In Japan, concepts of Western modern science are translated into Japanese words. A typical example is the English word "nature” being equated with the Japanese word "shizen.” However, this Japanese word normally refers to the supernatural as well as to natural things (Kawasaki , 1996; 2002). As a result, "shizen” may suggest the supernatural to pupils in the context of science lessons. In this way the Japanese language mode of science education leads pupils to misunderstand not only Western scientific concepts but also Western science. However, this problem is rarely realized amongst Japanese science educators who uncritically accept such translations. It is essential, therefore, to differentiate between "the Japanese language mode of science education” and genuine science education in which Western scientific concepts are taught correctly. By failing to do this, Japanese pupils are likely to fail to achieve a critical scientific literacy in which they are "learning science," "learning about science” and “doing science” (Hodson, 1998).

An important question arises in relation to the Japanese language mode of science education: how to draw Japanese science educators' attention to pupils' conceptual confusion originating from the Japanese translation. The notion of "language mode of science education” provides a powerful means for enabling science educators to differentiate their own language mode of science education from other language modes of science education; in other words, this notion offers a differentiating viewpoint. Depending on the non-SAE language used in science education, a differentiating viewpoint will remind science educators of potential conceptual confusion caused by translation.

Building on this earlier approach, with the help of axiomatics, this article presents a viewpoint for integrating various language modes of science education. An integrating viewpoint enables science educators to realize and reject the (implicit) claims to universality of Western modern science. Likewise, other language modes of science education will lose their rationales for claiming universality. Instead, all language modes of science education will acquire a new rationale - worldview education - which encourages pupils to foster their own language-culture 
identity whilst acquiring correct concepts of Western modern science.

Hereafter, the term "Western modern science" is abbreviated to "W-science" in the same way as Kawasaki (1996). There, “Western modern science” was termed "Western ethno-science,” and then abbreviated as “W-science.” Hence, the abbreviation indicates the relativistic view of W-science. In addition, the modifier "language-culture” is abbreviated to "L-C.” In the next section, "L-C incommensurability" is discussed briefly on the basis of my recent articles. Axiomatics is outlined in the third section, and a system of axioms about science education conducted in various L-C settings is proposed in the fourth section. The fifth section shows that the system of axioms encourages science educators as well as pupils to have a subjectivity-consciousness mind. The final section indicates that the subjectivity-consciousness mind will develop worldview education from science education conducted in various languages and that the subjectivity-consciousness mind will liberate pupils from "a prison of language.”

\section{A Worldview Incommensurate With Another}

This section illustrates L-C incommensurability, and will be helpful to the present discussion. The container-contents model of language views words as a container for their associated concepts. This model justifies removing the shizen-concept from "shizen" as the container and pouring the nature-concept into it (Kawasaki, 1996). In uttering “shizen,” science educators expect this Japanese word to convey correctly the nature-concept. This is what science educators automatically assume in the Japanese mode of science education. However, from a structural linguistics perspective, a word cannot be a container for its concepts, and so this model should be rejected (Kawasaki, 1996).

In a specific language (i.e., a system of words), a word cannot acquire its concept without having a relation to other words in the system. If the container-contents model were correct then a word could acquire its concept in a self-determining manner. Culler (1988) explains this by adducing the example of how the word "brown" acquires its concept within a system of colours.

Brown is what is not red, black, grey, yellow, etc., and the same holds for each of the 
other signifieds; When I utter the word green, such 'concept' as might be present is perhaps best represented as the combination of 'not-blue', 'not-red', 'not-yellow', etc. --a bundle of nots. The meaning of green is a space in an interpersonal network of differences. To give the meaning is not to recover something that was present.... (p.26; pp.112-113)

The green-concept is determined by a bundle of nots shared in a specific L-C community. It is important to compare two types of "green" in two different L-C communities. One L-C community articulates the rainbow in terms of seven colours whereas the other articulates it in terms of six colours. Even though the two greens are exactly the same in the sense of spectroscopy, they are not interchangeable for the reason that the two bundles of nots differ from each other.

In the same way, a word in a language is rarely directly interchangeable with a foreign word equated to it through translation because words acquire their concepts in bundles of nots. In acquiring its specific concepts every word refers eventually to all other words in the same language. Therefore, it does not make good sense to consider L-C phenomena without also considering the interrelationship among words. The notion of interrelationship is identical with “order” in the following citation from Suzuki, a distinguished Japanese linguist.

Man cannot come into direct contact with the elements composing his [sic] world as such. These elements constitute a world meaningless in itself, one which might aptly be described as disorderly and chaotic. One must conclude that the role of language is to bring order to this world and fashion in it meaningful and controllable objects, properties, and actions. (1993, p. 40)

The order a specific language brings to this world does not allow each word to acquire its concepts in a self-determining manner. Those people who speak a language as their first language are usually unaware that the order establishes a set of norms by which they can communicate. The set of norms is social and is properly called a worldview.

Being established as a way "to bring order to this world and fashion in it meaningful and controllable objects, properties, and actions,” worldview is woven into the language. At the same 
time, a value system, by which people live in their L-C community, comes into existence. This is the reason why "Facts are unalike to speakers whose language background provides for unlike formulations of them” (Whorf 1959, p. 235). In sharing the same language, people share an identical worldview. L-C incommensurability is experienced by persons who live by different worldviews.

It will be helpful in understanding $\mathrm{L}-\mathrm{C}$ incommensurability to imagine two L-C communities: the Duck community and the Rabbit community. In the Duck community people have never known rabbits, and in the Rabbit community people have never known ducks.

\section{(Figure 1 is here.)}

When a Duck person and a Rabbit person see Figure 1 at the same time, the Duck person's cognition is incommensurate with the Rabbit person's. The Duck person will regard it as a duck whereas the Rabbit person will regard it as a rabbit. The Duck person must see the protruding things as beaks, but the Rabbit person must see the same protruding things as ears. Because beaks and ears play different roles in their respective cognition, the Duck person cannot be in agreement with the Rabbit person. This is an example of L-C incommensurability between the two L-C communities.

As is well known, Jastrow (1900, p. 295) invented this figure, a shifting figure, in the context of gestalt psychology. Shifting figures "were originally used by gestalt psychologists in their attack on the constancy hypothesis, i.e., the claim that what we see is entirely determined by the retinal image” (Brown, 1979, pp.84-85). Obviously, gestalt psychologists presuppose that a single person experiences a gestalt shift, for which the necessary condition is that the person must know both ducks and rabbits beforehand. Thus, the two types of cognition -- duck and rabbit -- suddenly shift repeatedly from one to the other. I will not go further into gestalt psychology; rather, I will focus on the necessary condition.

Given the necessary condition for this gestalt shift, the duck and rabbit persons cannot experience such a gestalt shift. More importantly, the Duck people do not realize that they cannot 
imagine rabbits, nor do the Rabbit people realize that they cannot imagine ducks. Thus, L-C incommensurability originates from ignorance of this type: lack of knowledge about both what is known and what is unknown. Overcoming this lack of knowledge requires awareness of the theory of knowing (i.e., epistemology), in which science educators frame both knowledge and ignorance. From a critical constructivist perspective, Taylor (1998, p.1112) advocates science educators engaging pupils in epistemological reflection on the "social context" of their learning, because the knowledge and ignorance are societal. This is an assignment for those science educators who conduct non-SAE language modes of science education.

To clarify their task, imagine that the Duck and Rabbit communities correspond to SAE and non-SAE L-C communities, respectively. The Rabbit person can be likened to science educators whose first language is a non-SAE language. In the non-SAE language mode of science education, they teach $\mathrm{W}$-science to pupils whose first language is the same non-SAE language. These pupils' worldview differs from the W-scientific worldview woven into SAE languages to the same extent that the worldview shared in the Rabbit L-C community differs from that of the Duck L-C community. Although pupils unwittingly systematize their experience of natural phenomena in accordance with their worldview, very few science educators realize this difference in pupils’ cognition, especially science educators who uncritically accept the container-contents model. Moreover, science educators are likely to articulate their experience of natural phenomena in accordance with their own worldview.

Focusing on this difference in pupils' experience in the context of a social constructivist perspective on science teaching, Cobern (2000, p.6) explains the functional aspect of worldview: “a person's thinking is based on a set of first principles." He also defines: "worldview is the foundation upon which a person constructs cognitive and perceptual frameworks” (Cobern, 2000, p.9). Thus, a constructivist perspective on science teaching accepts a variety of worldviews as a set of first principles. However, in the end the constructivist perspective seems to emphasize only the W-scientific worldview for pupils' genuine understanding of W-science. Although the emphasis assures pupils' genuine understanding of W-science, they will be in danger of an L-C identity crisis in exchange for genuine understanding. This emphasis causes their conceptual confusion about the shizen-experience (or not nature-experience), in the Japanese language mode of science education. 
I have discussed pupils’ conceptual confusion caused by science teachers' unawareness in the Japanese language mode of science education. From the viewpoint of structural linguistics, this conceptual confusion can be illustrated in three ways. First, as discussed already, the Japanese term "shizen” refers to the supernatural (Kawasaki, 1996). Second, the Japanese word "kansatsu”, which is believed to be an equivalent of "to observe", encourages pupils to have mystical empathy with their objects in the science classroom; the closest possible English equivalent of "kansatsu" is “to contemplate” (Kawasaki, 1999; 2002).

And third, the Japanese word "jikken", which is believed to be an equivalent of "experiment," does not inspire pupils to form genuine $\mathrm{W}$-scientific laws due to an inherent lack of a dichotomy between the phenomenal world and the world of Ideas, to use the Platonic term (Kawasaki, 2001; 2002). Because this dichotomy is a characteristic inherent in SAE language and because the L-C incommensurability concerned with the three examples is closely related to the lack of the dichotomy in the Japanese language, it is highly probable that other non-SAE L-C settings for science education put pupils in similar states of conceptual confusion. Therefore, the present discussion is applicable to other non-SAE language modes of science education.

\section{Integrating Viewpoint}

In conducting epistemological reflection on a non-SAE language mode of science education, science educators have to realize an encounter with an SAE language mode of science education similar to the Rabbit person being confronted with the Duck culture. This leads science educators to establish a viewpoint differentiating between the non-SAE and the SAE language modes of science education. Therefore, the notion of "language mode of science education" is an archetypal example of the differentiating viewpoint. However, the differentiating viewpoint alone is insufficient for epistemological reflection. An integrating viewpoint is imperative. Without an integrating viewpoint, science educators would be inclined to judge one of the language modes of science education as supreme: most likely the SAE language mode of science education. These science educators unintentionally support scientism, that is, a belief in the superiority of W-science based on its (presumed) universality. 
In order to avoid this judgement about W-science, it is necessary to establish an integrating viewpoint which assures equitable treatment of all language modes of science education. The integrating viewpoint serves as a complement to the differentiating viewpoint. The relationship between these two viewpoints is similar to a genus-species relationship. The integrating viewpoint articulates a genus, whereas the differentiating viewpoint articulates different species belonging to the same genus. For instance, regarding the Duck/Rabbit shifting figure, the integrating viewpoint articulates the figure that is not yet interpreted as a duck or rabbit. The differentiating viewpoint finds the difference between the Duck and Rabbit types of cognition.

It is the integrating viewpoint that makes it possible to realize and discuss the difference, but the significance of an integrating viewpoint is usually overlooked. Against the backdrop of the integrating viewpoint, science educators will be able to investigate features of various language modes of science education in the same way that biologists investigate various species within a single genus. It is the integrating viewpoint that makes it possible to investigate various language modes of science education. Therefore, both the differentiating and integrating viewpoints should be equally illuminated.

Thus, it is essential to establish the integrating viewpoint in comparative studies in science education. However, comparative studies infrequently describe their integrating viewpoints. Comparative studies should give explicit descriptions of why science education in an L-C setting is comparable with that in another L-C setting. Since a possible integrating viewpoint is implicitly assumed, science educators are inclined to judge the L-C difference in science education only from SAE language modes of science education. This outlook on science education research is far removed from epistemological reflection and from achieving equity amongst various language modes of science education.

An integrating viewpoint will remind science educators about their outlook on science education research, and will help them to be aware of the backdrop against which comparative studies on language modes of science education is conducted. The backdrop of the integrating viewpoint offers a commonality for all language modes of science education, enabling science educators to describe L-C incommensurability. Thus, the integrating viewpoint also works as a framework within which all language modes of science education are articulated.

The essence of the integrating viewpoint is based on axiomatics, which gives a general 
description of geometry. As is well known, the axiomatic system of geometry makes it possible for mathematicians to think of various types of geometry -- Euclidean and non-Euclidean geometries -- “in the one” (Blanche, 1973, p.168). “The one” appears to be an axiomatic system, and is properly called "metageometry" that is a typical example of the integrating viewpoint. All types of geometry are constructed within this single axiomatic system. Thus, a genus-species relationship exists between "metageometry" and all types of geometry.

All types of geometry are actually constructed from combinations of the single axiomatic system and respective postulates. The following is a part of the axiom system of geometry.

If a point $P$ belongs to a line $L$, then we may say that $P$ lies on $L$ or that $L$ passes through $P$. Similarly, if $P$ belongs to a plane $E$, then we may say that $P$ lies in $E$ or that $E$ passes through P. (Moise, 1974, p.37)

It should be noticed that the terms "line," "point” and "plane” are indefinable; mathematicians are prohibited from finding any meaning in these indefinable terms. This is identical to the point that the intension of indefinable terms is nil and that their extension is unlimited. This is the essence of “integrating” in the context of the present discussion.

Instead, mathematicians focus their attention on the relationships among the indefinable terms appearing in the axioms.

[O]nly the relations are determined exactly and universally by the axioms, but nothing prevents the same system of relations from being able to support different systems of specific interpretations. (Blanche, 1973, p.168)

Since their extension is unlimited, it is possible to make various interpretations within a single axiomatic system. Accepting different interpretations of the same system of axioms, mathematicians construct different geometries. Mathematicians’ opinions of indefinable terms accords exactly with that of structural linguists: a concept of a word appears itself in a bundle of nots. The bundle of nots does nothing but establish the interrelationship among words in the language. 
Mathematicians make different interpretations of the single axiomatic system by combining it with different postulates. In a combination of the single axiom system and a postulate, indefinable terms acquire their meanings intrinsic in a specific geometry. Three typical postulates of "parallel” are shown in the following. They create the three geometries, the Euclidean, Lobachevskian and Reimannian, respectively.

The Euclidean Parallel Postulate: Given a line $L$ and a point $P$ not on $L$, there is one and only one line $L$ ' which contains $P$ and is parallel to $L$.

The Lobachevskian Parallel Postulate: Given a line $L$ and a point $P$ not on $L$, there are at least two lines $L ', L$ ” which contain $P$ and are parallel to $L$.

The Riemannian Parallel Postulate: No two lines in the same plane are ever parallel.

(Moise, 1974, pp.114-115)

In the present discussion, it is not necessary for those who are unfamiliar with geometry to understand what the foregoing means. The point critical to the present discussion is that the three postulates are definitely contradictory to each other (the underlined). Owing to this contradiction, corresponding theorems derived in different geometries are inevitably contradictory. For instance, "the sum of the angles of a triangle is equal to two right angles in Euclid's geometry, less than two right angles in that of Lobachevsky, and greater than two right angles in that of Riemann” (Poincare, 1952, p. 39). Even though the three types of triangle are incommensurate with each other, the reason they are comparable is that all geometries share the same axiom system as the integrating viewpoint.

It is meaningful to distinguish between two types of contradiction. One is about postulates and the other about theorems. The contradiction among theorems is derived from that among postulates. In the following, the contradiction among the theorems will be likened to L-C incommensurability, and the contradiction among the postulates will be likened to differences in worldview between L-C communities. In this way of distinguishing between the two types of contradiction, difference between language modes of science education will be derived from differences in worldview as “a set of first principles” (Cobern, 2000, p.6).

Consequently, if a proper axiomatic system is established, various language modes of science 
education will become comparable with each other on the basis of the overriding axiomatic system as the integrating viewpoint. Taking the integrating viewpoint into account, science educators will be able to ensure equity of language modes of science education in the same way that mathematicians ensure complete equity of all types of geometry. Obviously mathematicians think it ridiculous to give a specific geometry (e.g., Euclidian geometry) priority over others.

\section{Axiomatics Model of Science Education}

In the following, I propose a system of axioms regarding science education or all language modes of science education. This axiomatic system functions as the axiomatics model for investigating language modes of science education from the L-C equitable point of view. By means of the axiomatics model, it becomes possible to distinguish among the three stages of cognition - the axiom, postulate and theorem stages of cognition - in the same way that the general geometry distinguishes among geometrical axioms, postulates and theorems.

Axiom (1) is a possible axiom about "the world” in the sense of Suzuki (1993, p.40). The axiom includes indefinable terms [SCIENCE], [KNOWLEDGE] and [NATURE] at the axiom stage of cognition. To emphasize indefinable terms, I have expressed them in capital letters, and have put them in brackets.

[SCIENCE] is a system of [KNOWLEDGE] about [NATURE].

This establishes only the relationship among [SCIENCE], [KNOWLEDGE] and [NATURE], and describes nothing about actual L-C phenomena experienced in an L-C community.

When a language brings order to "his world as such" (Suzuki, 1993, p.40), a system of objects is articulated or created in the L-C community (Kawasaki, 1996). A worldview as a set of first principles begins working. In this way, and only when Axiom (1) is combined with a worldview at the postulate stage of cognition, the composite arranges for the L-C people to produce or articulate L-C phenomena inherent only in their L-C community. This is the birth of reality inherent in the L-C community. Thus, Axiom (1) will reveal the process of the birth of reality to science 
educators, and then will lead science educators to consider the L-C phenomena at the theorem stage of cognition. Accepting the process of the birth of reality, science educators will realize the three stages of cognition, that is, the axiom, postulate and theorem.

In addition to Axiom (1), "language mode of science education" is axiomatized. This offers an integrating viewpoint against which it becomes possible to compare various language modes of science education.

[SCIENCE EDUCATION] is a system of teaching [SCIENCE].

Axiom (2) includes a new indefinable term [SCIENCE EDUCATION] that has the extension of all language modes of science education, and forms an axiomatic system with Axiom (1) by sharing the same indefinable term [SCIENCE] with Axiom (1). Being combined with a specific postulate, the axiomatic system produces a language mode of science education and a science at the theorem stage of cognition.

This axiomatic system will stimulate science educators to conduct epistemological reflection on their language modes of science education if science educators take account of the process of the birth of reality. Then, their epistemological reflection will make it possible to conduct cross-cultural investigation of language modes of science education from an impartial point of view.

For example, in the Japanese language mode of science education, science educators will take the Japanese worldview into consideration realizing that the Japanese worldview is already woven into the Japanese language. In this perspective, a possible statement obtained from the axiomatic system is: The Japanese language mode of science education is a system of teaching W-science. This sentence at the theorem stage reveals what is usually done in the Japanese language mode of science education, and illustrates that the two different postulates are involved in it. One is the Japanese worldview postulated in the Japanese language mode of science education, and the other is the $\mathrm{W}$-scientific worldview in order to articulate $\mathrm{W}$-science at the theorem stage.

Confusion will be brought at the theorem stage, because these two worldviews are definitely inconsistent (Kawasaki, 2002). Science teachers in the Japanese language mode of science education are comparable to those mathematicians who could not distinguish Euclidean and 
non-Euclidean parallel postulates in geometry. The fictitious mathematicians would arbitrarily derive theorems from the two postulates. Due to the inconsistency between the two postulates, some theorems would be contradictory to others. However, the mathematicians could not identify the contradiction because of their confusing the two postulates. For the similar reason, science teachers tend to overlook contradictions at the theorem stage. This is the principal reason for conceptual confusion in pupils' minds in the Japanese language mode of science education. In order to prevent this confusion about worldviews as a postulate, science teachers need to differentiate carefully between the W-scientific and the Japanese worldviews, and elucidate the difference between them.

At the end of this section, I tabulate the six indefinable terms, namely, [LINE], [DUCK/RABBIT FIGURE], [LANGUAGE], [WORLD], [SCIENCE] and [SCIENCE EDUCATION]. Table 1 shows how each term acquires its actual meaning at the theorem stage of cognition. In the process of acquiring meanings, the relationship that the axiom establishes remains unchanged.

(Table 1 is here.)

In the table above, the indefinable term [DUCK/RABBIT FIGURE] may sound odd, because it is not a term but a figure. However, this figure plays the same role as indefinable terms for the reason that nobody is allowed to interpret the figure into anything at the axiom stage of cognition. And regarding [LANGUAGE], Kawasaki (1996) discusses how actual languages are organized by viewpoints on the basis of structural linguistics.

\section{Subjectivity-Consciousness in Science Education}

Normally, countries as political units establish their respective national education systems. Those countries are properly thought to be nation states. In each nation state, the use of the 
dominant language is generalized through its national education system. By means of the dominant language as a national language, each nation state has its national literature and history written in the national language (Kohn, 1973, p.324). Since a specific worldview is woven into the national language, it becomes possible to foster national identity in pupils' mind. They share the same worldview. This is the principal reason why every nation state claims responsibility for its national education. Although this idea of nation state is simplistic (e.g., omission of friction among sub-nations with different languages or worldviews in a nation state), every political unit models or intends to model the idea of nation state in the present time.

In addition to the three features above (i.e., a national language, literature and history), every nation state intends to introduce $\mathrm{W}$-science and technology.

[Thus] nationalism is closely linked ......, with the introduction of modern science and technology in the service of the nation, with the exaltation of the national language and traditions above the formerly frequent use of universal languages (in Europe Latin and later French) and universal traditions (Christianity and Islam). (Kohn, 1973, p.324)

This is the reason that science education (i.e., teaching W-science) is upheld globally and conducted in various languages. However, this gives rise to friction between worldviews in non-SAE language modes of science education.

In every non-SAE language mode of science education, pupils are led inevitably to conceptual confusion about W-scientific concepts or reality, as in the Japanese language mode of science education. There, science teachers are so enthusiastic about teaching W-science that they regard the non-SAE worldview, by which pupils acquire actual meanings in their L-C community, as impediments to pupils' learning of W-science. Science educators are inclined to emphasize the superiority of the W-scientific worldview to the non-SAE worldview concerned.

This outlook of science educators on the W-scientific worldview is inconsistent with the nation state objective of fostering national identity in pupils' minds. Therefore, the question of how to reconcile the $\mathrm{W}$-scientific and each non-SAE worldview should be considered in non-SAE language modes of science education. The reconciliation will start with science educators' realizing that pupils are experiencing conceptual confusion; then, their endeavour to eliminate the 
confusion will follow.

The reconciliation involves not only the $\mathrm{W}$-scientific but also the non-SAE worldviews, both of which are at the postulate stage of cognition. Therefore, it is necessary to differentiate these two worldviews; then, language modes of science education will be differentiated by means of the present axiomatic system. This leads science educators to a language related strategy for conducting non-SAE mode of science education: science education should be associated with foreign language education (Kawasaki, 1996). Foreign language educators make a deliberate differentiation between pupils' first language and the foreign language to be taught, and naturally differentiate the worldviews woven into the respective languages.

In science educators' differentiation, no sense of discrimination in the science classroom should be made. This is achieved with the aid of Axiom (1), which reminds them of the transcendental nature of worldview as a postulate. It should be emphasized that the worldview is transcendental: it lies beyond practical experience of the people in each L-C community. The worldview cannot be proven by facts or practical experience as can the L-C phenomena. To the contrary, these facts or practical experience become discernible on the basis of the worldview (Suzuki, 1993, p.40). This is very similar to the geometrical facts: any postulate cannot be proven by theorems.

Because the W-scientific worldview is not an exception (see Kawasaki, 2002), this transcendental nature of worldview makes it possible for science educators to relativize W-scientific worldview to all other worldviews. This is identical to ensuring the equity of sciences at the theorem stage of cognition. Those science educators who ensure equity will not replace the non-SAE worldview by the W-scientific worldview, but just contrast one with the other. Thus, science educators should keep the two worldviews in perspective in non-SAE language modes of science education.

This means that any cross-cultural study should be conducted at the postulate stage of cognition, not at the theorem stage. For example, I have elucidated the L-C incommensurability between "nature” and "shizen”, and have reduced this L-C incommensurability to the linguistic fact that only the English language (as a typical SAE language) has an established function to form abstract nouns (Kawasaki, 2002). This L-C incommensurability is revealed at the theorem stage whereas the lack of the established function in the Japanese language lies at the postulate 
state. The lack of the function does not mean a linguistic flaw (Kawasaki, 2002) but shows an identifying characteristic of the Japanese language. A worldview should not be judged with respect to another, because any worldview is transcendental by nature, as stated above. However, it is possible to compare with another worldview against the backdrop of Axiom (1).

The point is that, taking account of the contradiction between the two worldviews, science educators need to realize which worldview they now adopt in the science classroom. It is easy for foreign language teachers to realize which language they speak in foreign language education, but it is difficult for science teachers to realize which worldview they now adopt in non-SAE language modes of science education for the reason that the same language seems to be used. In order to accomplish this, science teachers need to develop a subjectivity-consciousness mind by conducting epistemological reflection. This entails cultivating their ability to make a choice between the W-scientific and their pupils' first worldviews and to realize the choice just made.

When science educators successfully develop this subjectivity-consciousness mind, their outlook on W-scientific objects will make a radical change from discovering them to choosing the W-scientific worldview within which W-scientific objects are articulated at the theorem stage. This change is identical with a departure from Platonism in the following sense.

Platonism as a philosophy of mathematics is the view that at least the most basic mathematical objects (e.g., real numbers, Euclidean squares) actually exist, independently of the human mind which conceives them. Their properties are discovered, not created. (Anglin \& Lambek, 1995, p.68)

Taking account of the similarity between mathematical objects and W-scientific reality in the sense of Platonic Ideal (Kawasaki, 2002), the subjectivity-consciousness mind will remind science educators that W-scientific reality is created at the theorem stage of cognition. Those science educators will realize which worldview is chosen in a non-SAE language mode of science education. Hence, L-C incommensurability will come under science educators’ control.

At the end of this section, I adduce two possible factors which make it difficult for science educators to realize the difference in worldviews at the postulate stage of cognition. One is the translation-origin factor due to the container-contents model of language (Kawasaki, 1996), as 
stated earlier. Because the container-contents model claims that language and worldview are separable, science educators tend to pay no attention to the difference in worldviews when translating W-scientific terms. In this process of translation, science educators unwittingly transform W-scientific concepts in accord with the non-SAE worldview, thereby contradicting the W-scientific worldview at the postulate stage. Consequently, the counterparts do not play the same role as in W-science at the theorem stage. As a result, the counterparts will inevitably deceive science educators and then their pupils (Kawasaki, 1996; 1999; 2002).

The other factor is many science educators' belief in the universality of W-science. The axiomatics model, which reveals the transcendental nature of worldview, will be useful for science educators' reassessing their belief. Usually, by emphasizing the universality of W-science in the Japanese language mode of science education, for example, science educators are inclined to think it right that the Japanese L-C worldview should be replaced by the $\mathrm{W}$-scientific worldview. The present axiomatics model will remind science educators that any worldview as a postulate should be treated equitably because of its transcendental nature. Those science educators who become conscious that the Japanese worldview should be neutral toward the W-scientific worldview will need a base of equitable comparison between them. The base is at the axiom stage of cognition, and the possible axiom system will consists of axioms (1) and (2).

\section{Toward Worldview Education}

Science educators with a subjectivity-consciousness mind will naturally develop the idea of worldview education from any type of language mode of science education. Then, worldview education will become an essential aspect of non-SAE language modes of science education. By focusing on differences between the two worldviews concerned, science educators keep them in perspective. These two worldviews are the $\mathrm{W}$-scientific worldview and the worldview woven into pupils’ first language. In worldview education, pupils will learn that every L-C reality, not excepting the $\mathrm{W}$-scientific reality, is created by a worldview beyond practical experience. And they will become able to reduce L-C incommensurability to the differences between the worldviews. Then, pupils will learn $\mathrm{W}$-science and their science, both of which are at the theorem 
stage, without any L-C prejudice.

As a result of worldview education, pupils will get the key to "a prison formed by the structural rules of our language” (Popper, 1994, p.52). Popper commented on this metaphor:

I am prepared to accept this metaphor, though I have to add to it that it is an odd prison as we are normally unaware of being imprisoned. We may become aware of it through culture clash. But then, this very awareness allows us to break out of the prison. If we try hard enough, we can transcend our prison by studying the new language and by comparing it with our own. (Popper, 1994, p.52)

Worldview education will be able to arrange "culture clash” for pupils. They will become aware of "being imprisoned," and will get the key to "the prison."

This means that drawing pupils’ attention to their conceptual confusion is identical to resolving their conceptual confusion. Those pupils who can get the key will successfully avoid their L-C identity crisis in the science classroom. Hence, science education will set forth a new objective in addition to $\mathrm{W}$-science teaching: worldview education wherein pupils learn $\mathrm{W}$-science with a subjectivity-consciousness mind by means of the axiomatics model. Worldview education will also liberate science teachers from "the cultural myths of modernist science, not through rejection, but, rather through critique that makes visible historical contingency” (Taylor, 1998, p.1121).

Pupils in worldview education will find themselves not obeying their worldview as a social norm but making an independent choice of their worldview at the postulate stage. Whether pupils obey or choose the results may be the same from the viewpoint of their learning $\mathrm{W}$-science. However, those pupils will regain their freedom to make a choice of worldview in non-SAE language modes of science education because they have the key to the prison of language, that is, worldview. They will not suppress the worldview woven into their first language.

Consequently, pupils will not replace their inherent worldview by the W-scientific worldview, and will find a difference in worldviews at the postulate stage. After reducing L-C incommensurability at the theorem stage to the difference between worldviews, pupils will become able to learn both sciences without prejudice. Thus, reconciliation will be brought about in 
pupils' minds. This means that both teaching W-science and fostering pupils' national identity will be accomplished in the science classroom. It will be confirmed that how to realize is synonymous with how to resolve.

Furthermore, the axiomatics model will stimulate science educators to undertake a mirror argument in the context of worldview education. A mirror argument always presupposes another one in which the first person and the second person are exchanged. They are mirror arguments to each other. For example, Kawasaki (2002) mounted an argument in which the author as a non-SAE language person discussed non-SAE language modes of science education against the backdrop of the W-scientific worldview. The mirror argument to Kawasaki (2002) will be: An author as an SAE language person discusses an SAE language mode of science education against the backdrop of a non-SAE language worldview.

In a pair of mirror arguments, an object of consideration will be exchanged as follows. From the viewpoint of a non-SAE language mode of science education, on the one hand, the W-scientific worldview is objective and corresponds to a foreign language in foreign language education. On the other hand, from the viewpoint of the SAE language mode of science education, the $\mathrm{W}$-scientific worldview is subjective and corresponds to the first language in foreign language education. The axiomatics model will make possible the pair of mirror arguments.

It may be concluded that a non-SAE type of worldview should be learnt equitably even in an SAE language mode of science education. Then, the pair of mirror arguments will be completed. If science educators pay more attention to worldviews woven into various non-SAE languages, a lot of pairs of mirror arguments will be made. Definitely this will bring great improvement in the fields of science education and science education research. Then, in worldview education conducted throughout the world, pupils will learn W-science and at least one non-SAE science. If science educators realize that $\mathrm{W}$-science is at the theorem stage of cognition, W-science will appear to regain universality to a certain extent.

\section{Acknowledgements}

This research is partly supported by Grant-in Aid for Scientific Research supported by the 
Ministry of Education, Culture, Sports, Science and Technology; the grant was given to a research project entitled: Reconciliation between the Japanese traditional worldview and science education. I would like to thank Dr. Loo Seng Piew for his helpful comments on Axiom (1). I also acknowledge valuable discussions in the International Joint Research on Culture, Language and Gender Sensitive Science Teacher Education Program, CLAGS-STEP (2003). Specifically, I am grateful to Dr. Glen Aikenhead for inspiring me to develop the discussion on mirror argument and to Dr. Peter Taylor for his helpful comments and polishing of the draft. It is my fortune that both of them are members of CLAGS-STEP (2003).

\section{Notes}

1. The term "SAE" is an abbreviation for "Standard Average European" defined in Language, Thought, and Reality (Whorf, 1959, p.138). The abbreviation stands for English, French, German, etc., for example. Those who use one of them as a first language (i.e., mother tongue) are SAE people. Consequently, there is the notion that "SAE" divides L-C communities into two: Western and non-Western communities (Kawasaki, 1996). Therefore, non-Western countries are those where people use non-SAE languages as their first language.

\section{References}

Anglin, W. S. \& Lambek, J. (1995). The Heritage of Thales. New York: Springer.

Blanche, R. (1973). Axiomatization. In P. P. Wiener (Editor in Chief), Dictionary of The History of Ideas Vol.I. New York: Charles Scriber’s Sons.

Brown, H. I. (1979). Perception, Theory and Commitment (Phoenix Edition). Chicago: The University of Chicago Press.

Cobern, W. W. (2000). Everyday Thoughts about Nature. Dordrecht: Kluwer.

Culler, J.: 1988[1976], Saussure (sixth impression), Fontana Press, London.

Jastrow, J. (1900). Fact and Fable in Psychology. Boston: Houghton Mifflin.

Kawasaki, K. (1996). The Concepts of Science in Japanese and Western Education. Science \& Education, 5(1), 1-20.

Kawasaki, K. (1999). A Deductive Description of Cultural Diversity of ‘Observation'. Journal of 
Science Education in Japan, 23(4), 258-270.

Kawasaki, K. (2001). An Aspect of 'Experiment' in Science Education in Japan. Kagakukyouiku Kenkyu (Journal of Science Education in Japan), 25(1), 2-10 (In Japanese).

Kawasaki, K. (2002). A Cross-Cultural Comparison of English and Japanese Linguistic Assumptions Pupils’ Learning of Science. Canadian and International Education, 31(1), 19-51.

Kohn, H. (1973). Nationalism. In P. P. Wiener (Editor in Chief), Dictionary of The History of Ideas Vol. III. New York: Charles Scriber’s Sons.

Hodson, D. (1998). Teaching and Learning Science. Buckingham: Open University Press

Moise, E. E. (1974). Elementary Geometry from an Advanced Standpoint (Second Edition). Massachusetts: Addison-Wesley Publishing.

Poincare, H. (1952). Science and Hypothesis. New York: Dover.

Popper, K. R. (1994). The Myth of the Framework. London: Routledge.

Suzuki, T. (1993). Words in Context, translated by A. Miura. Tokyo: Kodansha International.

Taylor, P. C. (1998). Constructivism: Value Added. In B. J. Fraser and K. G. Tobin (Editors), International Handbook of Science Education (pp. 1111-1123). Dordrecht: Kluwer Academic Publishers.

Whorf, B. L. (1959[1956]). Language, Thought, and Reality (fourth printing). New York: John Wiley and Sons. 
Figure 1

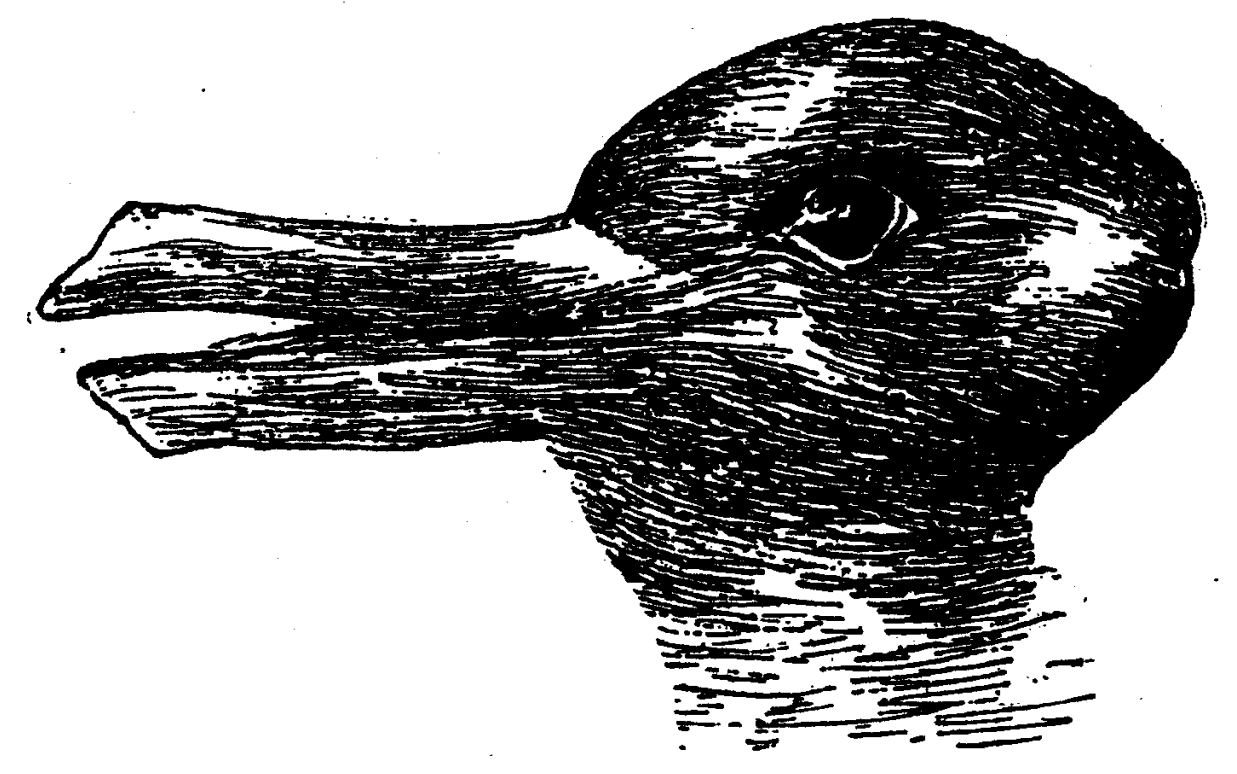

Fig. 1: Duck/Rabbit Figure (Shifting Figure) from Jastrow (1900, p.295) 
Table 1 Indefinable term, Postulate and Reality at the Theorem Stage of Cognition

\begin{tabular}{|c|c|c|}
\hline INDEFINABLE TERM & POSTULATE & REALITY AT THEOREM STAGE \\
\hline \hline \multirow{2}{*}[\mathrm{LINE}]{} & Euclidian parallel postulate & lines in the sense of Euclidean geometry \\
\cline { 2 - 3 } & Lobachevskian parallel postulate & lines in the sense of Lobachevskian geometry \\
\cline { 2 - 3 } & Riemannian parallel postulate & lines in the sense of Riemannian geometry \\
\hline \multirow{2}{*}{ DUCK/RABBIT FIGURE] } & Duck-Culture postulate & recognition of a duck as reality \\
\cline { 2 - 3 } & Rabbit-Culture postulate & recognition of a rabbit as reality \\
\hline$[$ LANGUAGE] & viewpoint to articulate words & languages \\
\hline [WORLD] & worldview & object systems \\
\hline [SCIENCE] & worldview woven into language & language modes of science education \\
\hline [SCIENCE EDUCATION] & worldview woven into L-C Setting & \\
\hline
\end{tabular}

